\title{
Stereoselective Syntheses of the Antihistaminic Drug Olopatadine and its $E$-isomer
}

Joan Bosch ${ }^{[a]}$, Jordi Bachs ${ }^{[b]}$, Antonia M. Gómez ${ }^{[a]}$, Rosa Griera ${ }^{[a]}$, Marta Écija ${ }^{[a]}$, and Mercedes Amat $^{[a]}$

${ }^{[a]}$ Laboratory of Organic Chemistry, Faculty of Pharmacy, and Institute of Biomedicine (IBUB), University of Barcelona, Av. Joan XXIII s/n, 08028 Barcelona ${ }^{[b]}$ Urquima, S.A., Arnau de Vilanova 22-42, 08105 Sant Fost de Campsentelles (Barcelona) joanbosch@ub.edu

RECEIVED DATE (will be automatically inserted after manuscript is accepted)

Practical stereoselective synthetic routes to the antihistaminic drug olopatadine and its $E$-isomer have been developed, the key steps being a trans stereoselective Wittig olefination using a nonstabilized phosphorus ylide and a stereoselective Heck cyclization. The stereoselectivity of the Wittig reaction depends on both the phosphonium salt anion and the cation present in the base used to generate the ylide.

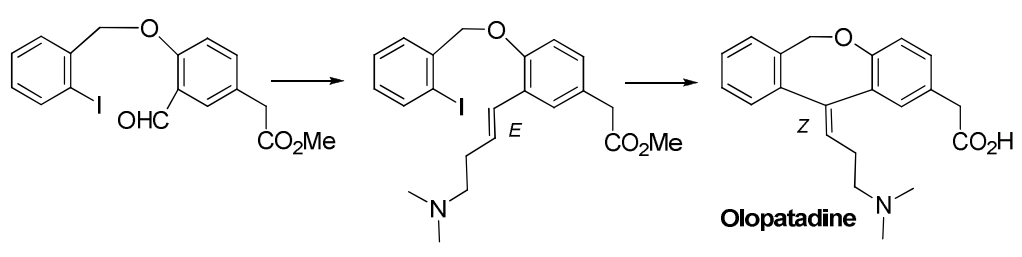

Olopatadine is a non-sedating histamine H1-receptor antagonist with mast cell stabilizing properties. This dual action allows seasonal allergic conjunctivitis and rhinitis signs and symptoms to be controlled. ${ }^{1}$ Structurally, olopatadine is a dibenzo[b,e]oxepin derivative bearing a $Z$-configurated (dimethylamino)propylidene substituent and an acetic acid chain at the C-11 and C-2 positions, respectively, of the tricyclic system. Previous synthetic approaches to olopatadine ${ }^{2}$ involve the 
generation of the trisubstituted exocyclic double bond from a tricyclic dibenz $[b, e]$ oxepin ketone, which represents a drawback in terms of $Z / E$ stereoselectivity. ${ }^{3}$

We herein report short and practical stereoselective syntheses of olopatadine and its $E$-isomer (transolopatadine), based on a common strategy involving successive stereoselective Wittig and intramolecular Heck reactions as the key steps. ${ }^{4}$ After a Williamson reaction to assemble the benzyl aryl ether moiety, the Wittig olefination would lead to the key intermediates $\mathbf{1}$ or $\mathbf{2}$, depending on the aromatic aldehyde and aryl iodide derivatives used as the starting materials (Scheme 1). Taking into account that in particular cases, by choosing the appropriate reaction conditions, high selectivity for $(E)$ and/or (Z)-alkene can be attained in the Wittig reaction of nonstabilized phosphorus ylides with aldehydes $^{5}$ and that the Heck reaction usually involves a $s y n$-addition/syn-elimination mechanism, ${ }^{6}$ the above Williamson-Wittig-Heck strategy could provide stereoselective access to both olopatadine and its E-isomer.

SCHEME 1. Synthetic Strategy. A Wittig/Heck Approach to Olopatadine and its E-isomer

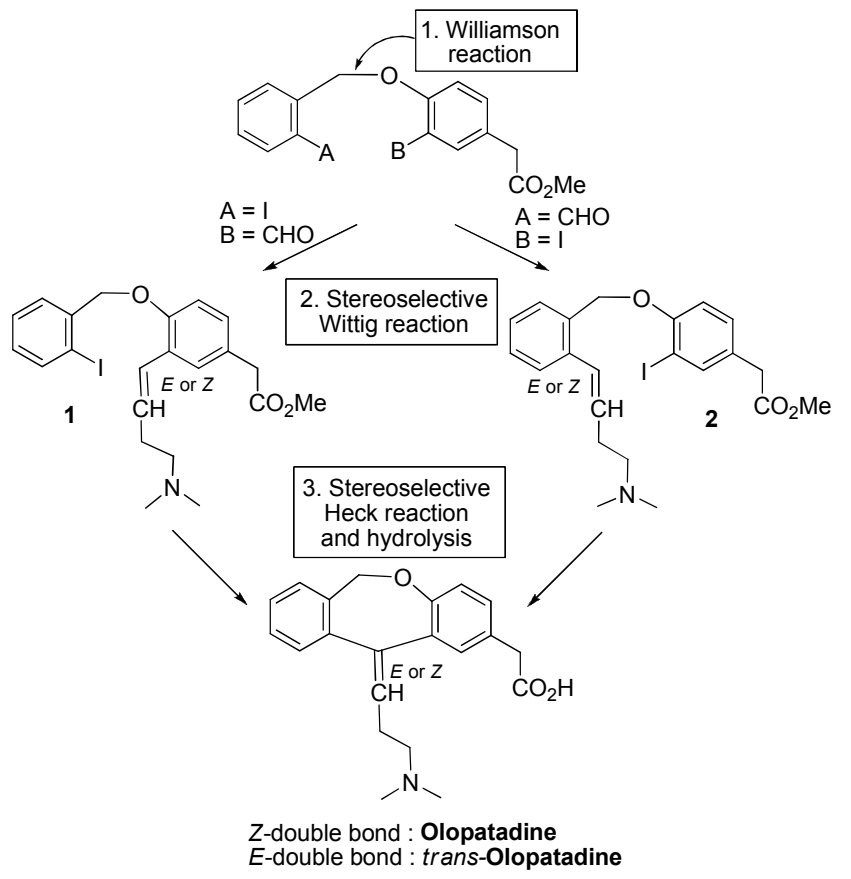


The starting aldehyde $\mathbf{5}$ was prepared from 2-iodobenzyl chloride $\mathbf{3}$ and 3-formyl-4hydroxyphenylacetic ester $4^{7}$ under the reaction conditions indicated in Scheme 2. The Wittig reaction from 5 was initially performed using the ylide generated from the commercially available phosphonium bromide $6(\mathrm{X}=\mathrm{Br})$ by treatment with KHMDS in toluene (Table 1 , entry 1$){ }^{8}$ giving a mixture of $E / Z$ alkenes in a 1:3 ratio. Similar results were obtained starting from the iodide salt $6(X=I){ }^{9}$ (entry 2$)$. Notably, the use of the lithium base LHMDS to generate the ylide from phosphonium iodide $6(X=I)$ produced a dramatic change in the stereoselectivity of the Wittig reaction, leading to a $9: 1 E / Z$ ratio of alkenes 1 in $73 \%$ yield (entry 3 ). ${ }^{10}$ A similar effect, although less pronounced (4:1 E/Z ratio), was observed from the bromide salt $6(X=B r)$ (entry 4). In contrast, the use of phosphonium chloride $6(X=$ $\mathrm{Cl})^{11}$ as the ylide precursor gave poor results in terms of both selectivity and chemical yield (entry 5).

\section{SCHEME 2. The Wittig Reaction from Aldehyde 5}
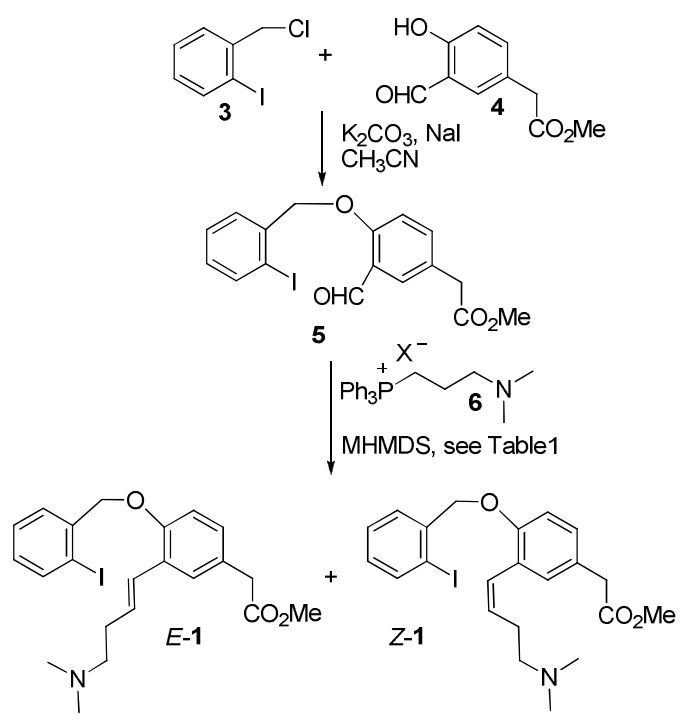
TABLE 1. Stereochemistry Dependence on the Cation in the Wittig Reaction ${ }^{a}$

\begin{tabular}{ccccc}
\hline entry & base, M & $\mathbf{6 , X}$ & $E / Z$ ratio $^{b}$ & $\mathbf{1 , \text { yield } ( \% )}$ \\
\hline 1 & $\mathrm{~K}$ & $\mathrm{Br}$ & $1: 3$ & 60 \\
2 & $\mathrm{~K}$ & $\mathrm{I}$ & $1: 3$ & $85^{c}$ \\
3 & $\mathrm{Li}$ & $\mathrm{I}$ & $9: 1$ & 73 \\
4 & $\mathrm{Li}$ & $\mathrm{Br}$ & $4: 1$ & $77^{c}$ \\
5 & $\mathrm{Li}$ & $\mathrm{Cl}$ & $1: 1.5$ & $37^{c}$
\end{tabular}

${ }^{a}$ Reaction conditions: aldehyde 5 (0.02 $\mathrm{M}$ in anh toluene), phosphonium salt 6 (4.2 equiv), MHMDS (4.2 equiv), rt.

${ }^{b}$ Calculated by ${ }^{1} \mathrm{H}$ NMR and HPLC.

${ }^{c}$ Calculated by HPLC from the crude reaction mixture.

The above trans selectivity is worthy of comment as Wittig olefination reactions of nonstabilized phosphorus ylides with aromatic aldehydes usually produce the thermodynamically less stable cis isomer as the major product. ${ }^{5}$ The presence of a nucleophilic group (for instance, amino) in the side chain of these ylides causes a shift in the stereochemistry of the alkene product towards the trans isomer, ${ }^{12}$ whose production is also increased by lithium ions. ${ }^{13}$

The pronounced effect of the lithium cation on alkene stereochemistry was also observed in the Wittig reaction from benzaldehyde 9 , which was prepared from 2 -formylbenzyl bromide $7^{14}$ and 4 hydroxy-3-iodophenylacetic ester $\mathbf{8}^{15}$ (Scheme 3). Thus, alkenes 2 were produced in an $E / Z$ ratio of 1:3 when the ylide was generated in toluene solution from phosphonium iodide $\mathbf{6}(\mathrm{X}=\mathrm{I})$ using KHMDS as the base (Table 2, entry 1). Neither the use of THF as the solvent (entry 2) nor starting from the bromide salt (entry 3 ) substantially modified the $E / Z$ ratio. However, when using the lithium base LHMDS to generate the ylide from iodide $6(\mathrm{X}=\mathrm{I})$, the stereoselectivity was reversed, with a significant enhancement of $E$ stereochemistry, leading to a $E / Z$ ratio of 3.5:1 (entry 4$)$. 


\section{SCHEME 3. The Wittig Reaction from Aldehyde 9}
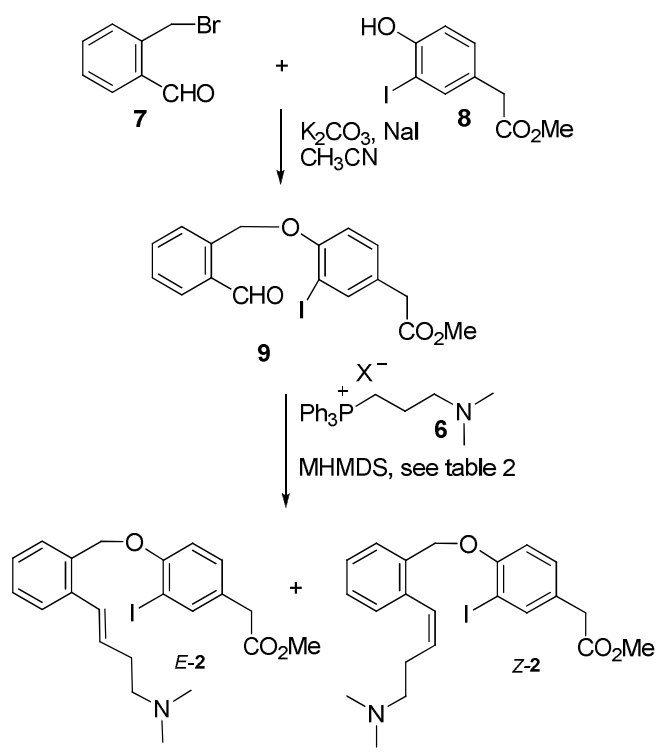

TABLE 2. Stereochemistry Dependence on the Cation in the Wittig Reaction ${ }^{a}$

\begin{tabular}{ccclcc}
\hline entry & base, $\mathrm{M}$ & $\mathbf{6 ,}, \mathrm{X}$ & solvent & $E / Z$ ratio $^{b}$ & $\mathbf{2 , ~ y i e l d ~}^{c}(\mathbf{\%})$ \\
\hline 1 & $\mathrm{~K}$ & $\mathrm{I}$ & toluene & $1: 3$ & 73 \\
2 & $\mathrm{~K}$ & $\mathrm{I}$ & $\mathrm{THF}$ & $1: 4$ & 64 \\
3 & $\mathrm{~K}$ & $\mathrm{Br}$ & toluene & $1: 3$ & 86 \\
4 & $\mathrm{Li}$ & $\mathrm{I}$ & toluene & $3.5: 1$ & 88
\end{tabular}

${ }^{a}$ Reaction conditions: aldehyde 9 (0.02 $\mathrm{M}$ in anh toluene or THF), phosphonium salt 6 (4.2 equiv), MHMDS (4.2 equiv), rt.

${ }^{b}$ Calculated by ${ }^{1} \mathrm{H}$ NMR and HPLC.

${ }^{c}$ Calculated by HPLC from the crude reaction mixture.

From the synthetic standpoint, with four different alkenes now in hand, prepared in acceptable yield and good $(E-1)$ to acceptable $(Z-1, E-2, Z$-2) stereoselectivity, we were ready to tackle the key Heck cyclization. The intramolecular Heck reaction from iodo alkene $E-\mathbf{1}$ was performed under solid-liquid phase transfer conditions, using a stoichiometric quantity of $\mathrm{Bu}_{4} \mathrm{NCl}$ as the transfer agent ${ }^{16}$ and $\mathrm{K}_{2} \mathrm{CO}_{3}$ as the base, ${ }^{17}$ in the presence of a catalytic amount of $\mathrm{Pd}(\mathrm{OAc})_{2}$ (without phosphine ligands ${ }^{18}$ ) in an 
acetonitrile-water mixture. ${ }^{19}$ A single dibenzoxepin derivative 10, bearing a Z-configurated double bond, was formed with complete stereoselectivity in $60 \%$ yield. A similar Pd-catalyzed cyclization from iodo alkene $E$-2 stereoselectively afforded $E$ dibenzoxepin $\mathbf{1 1}$ in $55 \%$ yield. The above results were not unexpected and are consistent with a syn-addition of the initially formed arylpalladium intermediate to the alkene, with a subsequent syn $\beta$-elimination of a hydridopalladium halide, as outlined in Scheme 4.

\section{SCHEME 4. Stereoselective Heck Reactions}

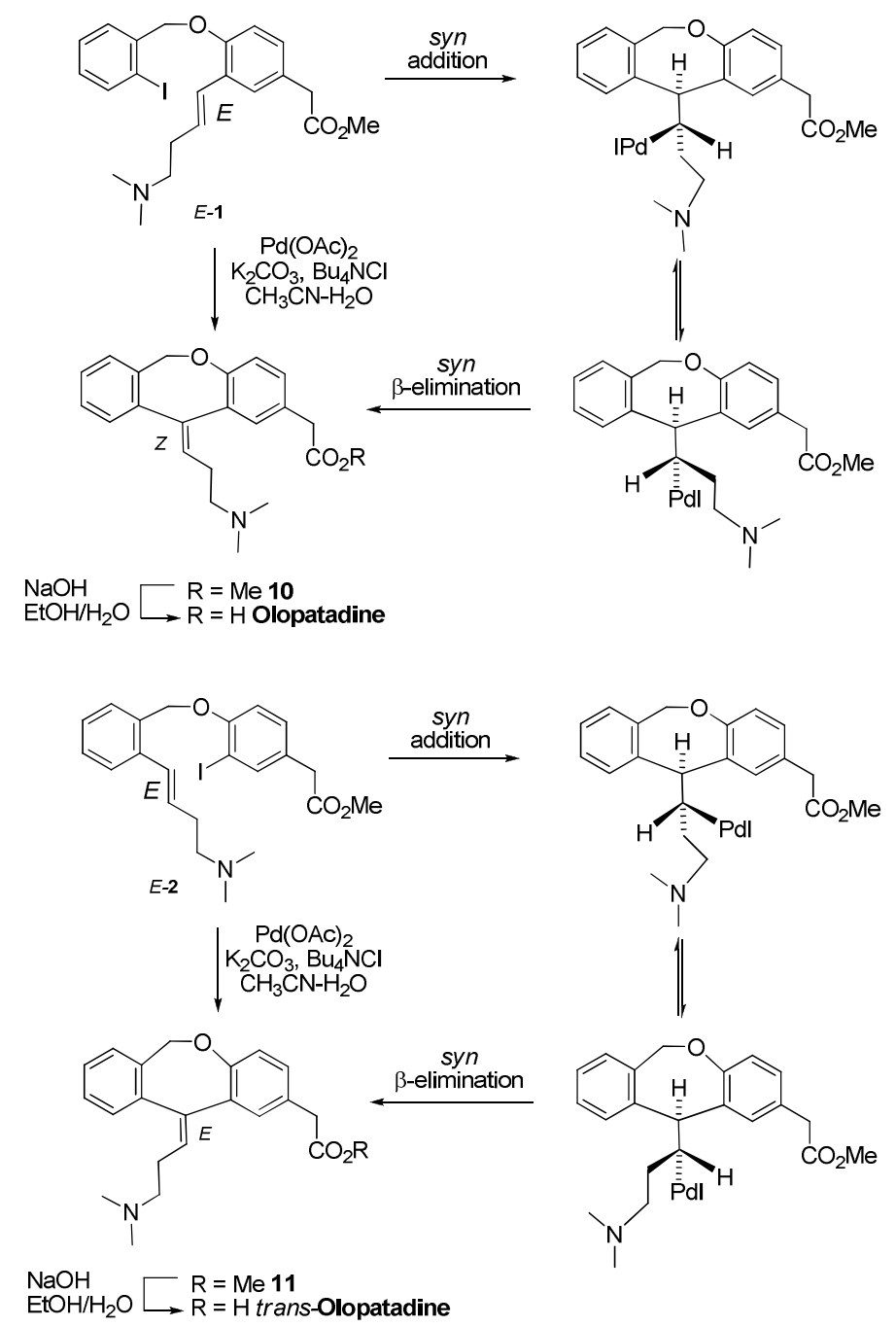


However, in contrast with the high stereoselectivity observed in the above Heck arylations from trans alkenes $E$-1 and $E-2$, the Heck cyclization from cis alkenes $Z-\mathbf{1}$ and $Z-2$ was not stereoselective, leading to mixtures of the cyclized products $\mathbf{1 0}$ and $\mathbf{1 1}$ (approximate ratios 3:2).

Finally, alkaline hydrolysis of the cyclized products $\mathbf{1 0}$ and $\mathbf{1 1}$ furnished the target drug olopatadine and its $E$-isomer, respectively.

In summary, we have developed practical synthetic routes to the antihistaminic drug olopatadine and its $E$-isomer based on a common strategy involving successive highly stereoselective Wittig olefination and Heck cyclization reactions as the key steps.

\section{Experimental Section}

(3-Dimethylaminopropyl)triphenylphosphonium Iodide $(6, X=I)$. A mixture of 3-(dimethylamino)1-propyl chloride hydrochloride (20 g, $126 \mathrm{mmol}), \mathrm{PPh}_{3}(33 \mathrm{~g}, 126 \mathrm{mmol})$, and $\mathrm{NaI}$ (19 g, $\left.126 \mathrm{~mol}\right)$ in acetonitrile $(80 \mathrm{~mL})$ was heated at reflux for 5.5 days. After cooling at $\mathrm{rt}, \mathrm{H}_{2} \mathrm{O}$-acetonitrile $(2: 1,360$ $\mathrm{mL}$ ) was added, and the mixture was stirred at $45{ }^{\circ} \mathrm{C}$ for $0.5 \mathrm{~h}$. The resulting suspension was filtered, solid $\mathrm{K}_{2} \mathrm{CO}_{3}$ was added to the filtrate until $\mathrm{pH}$ 9-10, and the solution was extracted with $\mathrm{CH}_{2} \mathrm{Cl}_{2}(3 \times 150$ $\mathrm{mL}$ ). The combined organic extracts were dried and concentrated, and the resulting oil was dissolved in $\mathrm{CH}_{2} \mathrm{Cl}_{2}(25 \mathrm{~mL})$. AcOEt $(120 \mathrm{~mL})$ was added, and the mixture was stirred until the formation of a white solid, which was collected by filtration (52 g, 87\%): mp 138-141 ${ }^{\circ} \mathrm{C} ;{ }^{1} \mathrm{H}$ NMR $\left(300 \mathrm{MHz}, \mathrm{CDCl}_{3}\right) \delta$ 1.75-1.87 (m, 2H), $2.13(\mathrm{~s}, 6 \mathrm{H}), 2.54(\mathrm{t}, J=5.9 \mathrm{~Hz}, 2 \mathrm{H}), 3.65-3.75(\mathrm{~m}, 2 \mathrm{H}), 7.70-7.87(\mathrm{~m}, 15 \mathrm{H}) ;{ }^{13} \mathrm{C}$ NMR (75.4 MHz, $\left.\mathrm{CDCl}_{3}\right) \delta 20.4(\mathrm{~d}, J=52.2 \mathrm{~Hz}), 20.5(\mathrm{~d}, J=3.3 \mathrm{~Hz}), 45.2(\mathrm{~s}), 58.2(\mathrm{~d}, J=16.0 \mathrm{~Hz})$, $117.9(\mathrm{~d}, J=86.2 \mathrm{~Hz}), 130.3(\mathrm{~d}, J=12.6 \mathrm{~Hz}), 133.3(\mathrm{~d}, J=10.5 \mathrm{~Hz}), 134.9$ (d, $J=2.7 \mathrm{~Hz}) ; \mathrm{IR}(\mathrm{KBr})$ 995, 1111, $1438 \mathrm{~cm}^{-1}$. Anal. Calcd for $\mathrm{C}_{23} \mathrm{H}_{27} \mathrm{NIP}: \mathrm{C}, 58.11 ; \mathrm{H}, 5.73 ; \mathrm{N}, 2.95$; I, 26.70. Found: C, 58.13; H, 5.66; N, 2.90; I, 26.58. 
(3-Dimethylaminopropyl)triphenylphosphonium Chloride $(6, \mathbf{X}=\mathbf{C l})$. A mixture of 3(dimethylamino)-1-propyl chloride hydrochloride $(10 \mathrm{~g}, 63 \mathrm{mmol})$ and $\mathrm{PPh}_{3}(16.5 \mathrm{~g}, 63 \mathrm{mmol})$ in 1butanol $(40 \mathrm{~mL})$ was heated at reflux for 4 days. The mixture was cooled at rt, toluene- $\mathrm{Et}_{2} \mathrm{O}(1: 1,50$ $\mathrm{mL}$ ) was added, and the solution was kept in the refrigerator for a night. The solid formed was filtered and washed with toluene- $\mathrm{Et}_{2} \mathrm{O}$ and $\mathrm{Et}_{2} \mathrm{O}$ to give pure $6 \cdot \mathrm{HCl}(\mathrm{X}=\mathrm{Cl})(15.6 \mathrm{~g}, 59 \%)$ as a white powder. A solution of this hydrochloride in $2 \mathrm{~N}$ aqueous $\mathrm{K}_{2} \mathrm{CO}_{3}(50 \mathrm{~mL})$ was extracted with $\mathrm{CH}_{2} \mathrm{Cl}_{2}(3 \times 30 \mathrm{~mL})$. The combined organic extracts were dried and concentrated to give an oil, which was cooled in the refrigerator for 2 days to afford $6,(\mathrm{X}=\mathrm{Cl})$ as a hygroscopic white solid $(12.4 \mathrm{~g}, 90 \%)$ : $\mathrm{mp} 188-191{ }^{\circ} \mathrm{C}$; ${ }^{1} \mathrm{H}$ NMR $\left(300 \mathrm{MHz}, \mathrm{CDCl}_{3}\right) \delta$ 1.74-1.87 (m, 2H), $2.12(\mathrm{~s}, 6 \mathrm{H}), 2.53(\mathrm{t}, J=6.0 \mathrm{~Hz}, 2 \mathrm{H}), 3.77-3.89$ (m, 2H), 7.69-7.89 (m, 15H); ${ }^{13} \mathrm{C}$ NMR (75.4 MHz, $\left.\mathrm{CDCl}_{3}\right) \delta 19.9(\mathrm{~d}, J=52.2 \mathrm{~Hz}), 20.5(\mathrm{~d}, J=3.3 \mathrm{~Hz})$, 45.0 (s), 58.2 (d, $J=16.0 \mathrm{~Hz}), 118.0$ (d, $J=86.2 \mathrm{~Hz}), 130.2$ (d, $J=12.6 \mathrm{~Hz}), 133.2(\mathrm{~d}, J=9.9 \mathrm{~Hz})$, $134.7\left(\mathrm{~d}, J=2.8 \mathrm{~Hz}\right.$ ); IR (KBr) 995, $1112,1434 \mathrm{~cm}^{-1}$. Anal. Calcd for $\mathrm{C}_{23} \mathrm{H}_{27} \mathrm{NClP}: \mathrm{C}, 71.96$; H, 7.09; N, 3.65; Cl, 9.24. Found: C, 71.93; H, 7.15; N, 3.52; Cl, 9.45 .

Methyl [3-Formyl-4-(2-iodobenzyloxy)phenyl]acetate (5). A solution of methyl (3-formyl-4hydroxyphenyl)acetate $(16.7 \mathrm{~g}, 85.9 \mathrm{mmol})$ in acetonitrile $(68 \mathrm{~mL})$ was slowly added at $\mathrm{rt}$ to a mixture of 1-(chloromethyl)-2-iodobenzene $(21.7 \mathrm{~g}, 85.9 \mathrm{mmol}), \mathrm{K}_{2} \mathrm{CO}_{3}(13.1 \mathrm{~g}, 94.5 \mathrm{mmol})$, and $\mathrm{NaI}(3.22 \mathrm{~g}$, $21.5 \mathrm{mmol})$ in acetonitrile $(273 \mathrm{~mL})$, and the mixture was heated at reflux for $3 \mathrm{~h}$. The solid was separated by filtration and the filtrate was concentrated. The resulting residue was dissolved in toluene (330 mL), and the organic solution was washed with $0.1 \mathrm{~N}$ aqueous $\mathrm{NaOH}$ and $\mathrm{H}_{2} \mathrm{O}$, dried, and concentrated. The residue was dissolved in acetone $(330 \mathrm{~mL})$ and poured into $\mathrm{H}_{2} \mathrm{O}(500 \mathrm{~mL})$. The mixture was filtered and the resulting solid was crystallized from toluene-cyclohexane to afford 5 (24.7 g, 70\%): mp 68-70 ${ }^{\circ} \mathrm{C} ;{ }^{1} \mathrm{H}$ NMR (300 MHz, $\left.\mathrm{CDCl}_{3}\right) \delta 3.61(\mathrm{~s}, 2 \mathrm{H}), 3.70$ (s, 3H), 5.15 (s, 2H), 7.00-7.10 $(\mathrm{m}, 2 \mathrm{H}), 7.37-7.42(\mathrm{~m}, 1 \mathrm{H}), 7.48-7.51(\mathrm{~m}, 2 \mathrm{H}), 7.77(\mathrm{~d}, J=2.1 \mathrm{~Hz}, 1 \mathrm{H}), 7.90(\mathrm{dd}, J=7.8,1.2 \mathrm{~Hz}, 1 \mathrm{H})$, $10.55(\mathrm{~s}, 1 \mathrm{H}) ;{ }^{13} \mathrm{C}$ NMR $\left(75.4 \mathrm{MHz}, \mathrm{CDCl}_{3}\right) \delta 39.9\left(\mathrm{CH}_{2}\right), 52.1\left(\mathrm{CH}_{3}\right), 74.4\left(\mathrm{CH}_{2}\right), 97.2(\mathrm{C}), 113.3$ 
$(\mathrm{CH}), 124.9(\mathrm{C}), 126.9(\mathrm{C}), 128.4(\mathrm{CH}), 128.6(\mathrm{CH}), 129.2(\mathrm{CH}), 129.8(\mathrm{CH}), 136.6(\mathrm{CH}), 138.0(\mathrm{C})$, $139.4(\mathrm{CH}), 159.6(\mathrm{C}), 171.5(\mathrm{C}), 189.2(\mathrm{CH})$; IR (NaCl) 1158, 1249, 1683, 1736, 2700, $2900 \mathrm{~cm}^{-1}$. Anal. Calcd for $\mathrm{C}_{17} \mathrm{H}_{15} \mathrm{IO}_{4}$ : C, 49.78; H, 3.69. Found: C, 49.67; H, 3.66.

Methyl [4-(2-Formylbenzyloxy)-3-iodophenyl]acetate (9). Operating as above, from 2(bromomethyl)benzaldehyde (11 g, $55.3 \mathrm{mmol})$, methyl (4-hydroxy-3-iodophenyl)acetate (16.1 g, 55 $\mathrm{mmol}), \mathrm{K}_{2} \mathrm{CO}_{3}(8.36 \mathrm{~g}, 60.5 \mathrm{mmol})$, and $\mathrm{NaI}(2.07 \mathrm{~g}, 13.8 \mathrm{mmol})$ in acetonitrile $(220 \mathrm{~mL})$, compound 9 (18 g, 80\%) was obtained after crystallization from acetone- $\mathrm{H}_{2} \mathrm{O}: \mathrm{mp} 93-97{ }^{\circ} \mathrm{C} ;{ }^{1} \mathrm{H} \mathrm{NMR}(300 \mathrm{MHz}$, $\left.\mathrm{CDCl}_{3}\right) \delta 3.54(\mathrm{~s}, 2 \mathrm{H}), 3.70(\mathrm{~s}, 3 \mathrm{H}), 5.55(\mathrm{~s}, 2 \mathrm{H}), 6.90(\mathrm{~d}, J=8.4 \mathrm{~Hz}, 1 \mathrm{H}), 7.46(\mathrm{dd}, J=8.2,2.2 \mathrm{~Hz}$, 1H), $7.54(\mathrm{dd}, J=7.8,7.5 \mathrm{~Hz}, 1 \mathrm{H}), 7.67-7.74(\mathrm{~m}, 2 \mathrm{H}), 7.88(\mathrm{dd}, J=6.0,1.3 \mathrm{~Hz}, 1 \mathrm{H}), 8.08(\mathrm{~d}, J=7.8$ $\mathrm{Hz}, 1 \mathrm{H}), 10.15(\mathrm{~s}, 1 \mathrm{H}) ;{ }^{13} \mathrm{C} \mathrm{NMR}\left(75.4 \mathrm{MHz}, \mathrm{CDCl}_{3}\right) \delta 39.6\left(\mathrm{CH}_{2}\right), 52.1\left(\mathrm{CH}_{3}\right), 68.6\left(\mathrm{CH}_{2}\right), 86.4(\mathrm{C})$, $112.3(\mathrm{CH}), 127.4(\mathrm{CH}), 127.7(\mathrm{CH}), 128.4(\mathrm{C}), 130.4(\mathrm{CH}), 132.4(\mathrm{C}), 134.2(\mathrm{CH}), 134.5(\mathrm{CH}), 138.8$ (C), $140.0(\mathrm{CH}), 156.0(\mathrm{C}), 171.6(\mathrm{C}), 193.4(\mathrm{CH})$; IR (KBr) 1252, 1691, 1731, 2750, $2833 \mathrm{~cm}^{-1}$. Anal. Calcd for $\mathrm{C}_{17} \mathrm{H}_{15} \mathrm{IO}_{4}$ : C, 49.78; H, 3.69; I, 30.94. Found: C, 49.81; H, 3.53; I, 31.24 .

Methyl 3-[4-(Dimethylamino)but-1-enyl]-4-(2-iodobenzyloxy)phenylacetate (1). LHMDS (1 M in THF, $51.5 \mathrm{~mL}, 51.5 \mathrm{mmol})$ was added dropwise to a suspension of $\mathbf{6}(\mathrm{X}=\mathrm{I})(24.3,51.2 \mathrm{mmol})$ in anhydrous toluene $(300 \mathrm{~mL})$, and the resulting mixture was stirred at $\mathrm{rt}$ for $1 \mathrm{~h}$. A solution of $5(5.00 \mathrm{~g}$, $12.2 \mathrm{mmol})$ in anhydrous toluene $(170 \mathrm{~mL})$ was added and the resulting mixture was stirred at $\mathrm{rt}$ for 2.5 h. The reaction was quenched with $\mathrm{H}_{2} \mathrm{O}(150 \mathrm{~mL})$, and the aqueous layer was extracted with toluene. The combined organic extracts were extracted with $2 \mathrm{~N}$ aqueous $\mathrm{HCl}(150 \mathrm{~mL})$, and then solid $\mathrm{K}_{2} \mathrm{CO}_{3}$ was added to the aqueous phase until $\mathrm{pH}$ 9. The alkaline solution was extracted with EtOAc, and the extracts were dried and concentrated. Column chromatography $\left(\mathrm{Al}_{2} \mathrm{O}_{3}\right.$, hexane-EtOAc 95:5 to 75:25) afforded compound 1 (4.26 g, 73\%; E/Z 9:1) as an oil. Pure samples of both isomers were obtained after additional column chromatography. 
Data for $\boldsymbol{E}-1:{ }^{1} \mathrm{H}$ NMR $\left(300 \mathrm{MHz}, \mathrm{CDCl}_{3}\right) \delta 2.27(\mathrm{~s}, 6 \mathrm{H}), 2.39-2.45(\mathrm{~m}, 4 \mathrm{H}), 3.55(\mathrm{~s}, 2 \mathrm{H}), 3.68(\mathrm{~s}, 3 \mathrm{H})$, $5.04(\mathrm{~s}, 2 \mathrm{H}), 6.18-6.29(\mathrm{~m}, 1 \mathrm{H}), 6.81(\mathrm{~d}, J=8.7 \mathrm{~Hz}, 1 \mathrm{H}), 6.84(\mathrm{~d}, J=16.2 \mathrm{~Hz}, 1 \mathrm{H}), 6.99-7.09$ (m, 2H), 7.34-7.39 (m, 2H), 7.50 (dd, $J=7.5,1.8 \mathrm{~Hz}, 1 \mathrm{H}), 7.86(\mathrm{dd}, J=7.9,1.2 \mathrm{~Hz}, 1 \mathrm{H}) ;{ }^{13} \mathrm{C} \mathrm{NMR}(75.4 \mathrm{MHz}$, $\left.\mathrm{CDCl}_{3}\right) \delta 31.8\left(\mathrm{CH}_{2}\right) ; 40.4\left(\mathrm{CH}_{2}\right), 45.4\left(2 \mathrm{CH}_{3}\right) ; 52.0\left(\mathrm{CH}_{3}\right), 59.4\left(\mathrm{CH}_{2}\right), 74.4\left(\mathrm{CH}_{2}\right), 96.9(\mathrm{C}), 112.8$ $(\mathrm{CH}), 125.1(\mathrm{CH}), 126.6(\mathrm{C}), 127.2(\mathrm{C}), 127.3(\mathrm{CH}), 128.3(\mathrm{CH}), 128.4(\mathrm{CH}), 128.6(\mathrm{CH}), 129.3(\mathrm{CH})$, $129.4(\mathrm{CH}), 139.1(\mathrm{CH}), 139.2(\mathrm{C}), 154.1(\mathrm{C}), 172.2(\mathrm{C})$; IR $(\mathrm{NaCl})$ 1245, $1737 \mathrm{~cm}^{-1}$. Anal. Calcd for $\mathrm{C}_{22} \mathrm{H}_{26} \mathrm{NIO}_{3}:$ C, 55.12; H, 5.47; N, 2.92. Found: C, 55.12; H, 5.48; N, 2.83.

Data for Z-1: ${ }^{1} \mathrm{H}$ NMR $\left(300 \mathrm{MHz}, \mathrm{CDCl}_{3}\right) \delta 2.22(\mathrm{~s}, 6 \mathrm{H}), 2.36-2.51(\mathrm{~m}, 4 \mathrm{H}), 3.57(\mathrm{~s}, 2 \mathrm{H}), 3.68(\mathrm{~s}, 3 \mathrm{H})$, $5.02(\mathrm{~s}, 2 \mathrm{H}), 5.74(\mathrm{dt}, J=11.4,6.8 \mathrm{~Hz}, 1 \mathrm{H}), 6.67(\mathrm{~d}, J=11.4 \mathrm{~Hz}, 1 \mathrm{H}) ; 6.84(\mathrm{~d}, J=8.7 \mathrm{~Hz}, 1 \mathrm{H}), 6.97-$ $7.13(\mathrm{~m}, 2 \mathrm{H}), 7.20(\mathrm{~d}, J=1.8 \mathrm{~Hz}, 1 \mathrm{H}), 7.32-7.40(\mathrm{~m}, 1 \mathrm{H}), 7.48-7.51(\mathrm{~m}, 1 \mathrm{H}), 7.84(\mathrm{dd}, J=7.8,1.2 \mathrm{~Hz}$, $1 \mathrm{H}) ;{ }^{13} \mathrm{C}$ NMR $\left(75.4 \mathrm{MHz}, \mathrm{CDCl}_{3}\right) \delta 27.1\left(\mathrm{CH}_{2}\right), 40.4\left(\mathrm{CH}_{2}\right), 45.3\left(2 \mathrm{CH}_{3}\right), 52.0\left(\mathrm{CH}_{3}\right), 59.4\left(\mathrm{CH}_{2}\right), 74.2$ $\left(\mathrm{CH}_{2}\right), 96.7(\mathrm{C}), 112.3(\mathrm{CH}), 125.2(\mathrm{CH}), 126.0(\mathrm{C}), 127.0(\mathrm{C}), 128.3(2 \mathrm{CH}), 128.7(\mathrm{CH}), 129.3(\mathrm{CH})$, $130.4(\mathrm{CH}), 130.9(\mathrm{CH}), 139.0(\mathrm{CH}), 139.2(\mathrm{C}), 154.9(\mathrm{C}), 172.1(\mathrm{C})$; IR (NaCl) 1244, $1738 \mathrm{~cm}^{-1}$. Anal. Calcd for $\mathrm{C}_{22} \mathrm{H}_{26} \mathrm{NIO}_{3}$ : C, 55.12; H, 5.47; N, 2.92. Found: C, 55.17; H, 5.54; N, 2.87.

Methyl 4-\{2-[4-(Dimethylamino)but-1-enyl]benzyloxy\}-3-iodophenylacetate (2). Operating as above, from aldehyde 9, under the reaction conditions of Table 2, mixtures of E-2 and Z-2 were obtained. Column chromatography $\left(\mathrm{Al}_{2} \mathrm{O}_{3}\right.$, hexane-EtOAc 95:5 to 75:25) afforded the pure isomers as oils.

Data for $\boldsymbol{E}$-2: ${ }^{1} \mathrm{H}$ NMR $\left(300 \mathrm{MHz}, \mathrm{CDCl}_{3}\right) \delta 2.27(\mathrm{~s}, 6 \mathrm{H}), 2.41-2.44(\mathrm{~m}, 4 \mathrm{H}), 3.52(\mathrm{~s}, 2 \mathrm{H}), 3.69$ (s, 3H), $5.14(\mathrm{~s}, 2 \mathrm{H}), 6.08-6.19(\mathrm{~m}, 1 \mathrm{H}), 6.72(\mathrm{~d}, J=15.6 \mathrm{~Hz}, 1 \mathrm{H}), 6.83(\mathrm{~d}, J=8.4 \mathrm{~Hz}, 1 \mathrm{H}), 7.18-7.31(\mathrm{~m}, 3 \mathrm{H})$, 7.46-7.53 (m, 2H), $7.71(\mathrm{~d}, J=2.1 \mathrm{~Hz}, 1 \mathrm{H}) ;{ }^{13} \mathrm{C} \mathrm{NMR}\left(75.4 \mathrm{MHz}, \mathrm{CDCl}_{3}\right) \delta 31.8\left(\mathrm{CH}_{2}\right), 39.6\left(\mathrm{CH}_{2}\right)$, $45.5\left(2 \mathrm{CH}_{3}\right), 52.1\left(\mathrm{CH}_{3}\right), 59.3\left(\mathrm{CH}_{2}\right), 69.4\left(\mathrm{CH}_{2}\right), 86.7(\mathrm{C}), 112.5(\mathrm{CH}), 126.1(\mathrm{CH}), 127.0(\mathrm{CH}), 127.5$ $(\mathrm{CH}), 128.2(\mathrm{CH}), 128.2(\mathrm{C}), 128.4(\mathrm{CH}), 130.2(\mathrm{CH}), 131.5(\mathrm{CH}), 132.6(\mathrm{C}), 136.6(\mathrm{C}), 140.1(\mathrm{CH})$, 156.3 (C), 171.7 (C); IR (NaCl) 1249, 1488, $1738 \mathrm{~cm}^{-1}$. Anal. Calcd for $\mathrm{C}_{22} \mathrm{H}_{26} \mathrm{NIO}_{3}$ : C, 55.12; H, 5.47; I, 26.47; N, 2.92. Found: C, 54.97; H, 5.43; I, 26.63; N, 2.85. 
Data for Z-2: ${ }^{1} \mathrm{H}$ NMR $\left(300 \mathrm{MHz}, \mathrm{CDCl}_{3}\right) \delta 2.17(\mathrm{~s}, 6 \mathrm{H}), 2.29-2.35(\mathrm{~m}, 4 \mathrm{H}), 3.51(\mathrm{~s}, 2 \mathrm{H}), 3.68(\mathrm{~s}, 3 \mathrm{H})$, $5.05(\mathrm{~s}, 2 \mathrm{H}), 5.76-5.85(\mathrm{~m}, 1 \mathrm{H}), 6.60(\mathrm{~d}, J=11.40 \mathrm{~Hz}, 1 \mathrm{H}), 6.78(\mathrm{~d}, J=8.1 \mathrm{~Hz}, 1 \mathrm{H}), 7.16-7.31(\mathrm{~m}, 4 \mathrm{H})$, 7.63-7.66 (m, 1H), $7.70(\mathrm{~d}, J=2.4 \mathrm{~Hz}, 1 \mathrm{H}) ;{ }^{13} \mathrm{C} \mathrm{NMR}\left(75.4 \mathrm{MHz}, \mathrm{CDCl}_{3}\right) \delta 26.9\left(\mathrm{CH}_{2}\right), 39.6\left(\mathrm{CH}_{2}\right)$, $45.3\left(2 \mathrm{CH}_{3}\right), 52.0\left(\mathrm{CH}_{3}\right), 59.3\left(\mathrm{CH}_{2}\right), 68.9\left(\mathrm{CH}_{2}\right), 86.6(\mathrm{C}), 112.2(\mathrm{CH}), 127.1(\mathrm{CH}), 127.4(2 \mathrm{CH}), 127.5$ $(\mathrm{CH}), 128.1(\mathrm{C}), 129.1(\mathrm{CH}), 130.1(\mathrm{CH}), 131.8(\mathrm{CH}), 134.0(\mathrm{C}), 135.6(\mathrm{C}), 140.0(\mathrm{CH}), 156.3(\mathrm{C})$, 171.6 (C); IR (NaCl) 1250, 1489, $1738 \mathrm{~cm}^{-1}$. Anal. Calcd for $\mathrm{C}_{22} \mathrm{H}_{26} \mathrm{NIO}_{3}$ : C, 55.12; H, 5.47; I, 26.47; N, 2.92. Found: C, 55.21; H, 5.68; I, 25.16; N, 2.87.

(Z)-Methyl 11-[3-(Dimethylamino)propylidene]-6,11-dihydrodibenz[b,e]oxepin-2-acetate (10). A mixture of the compound of $E-\mathbf{1}(600 \mathrm{mg}, 1.25 \mathrm{mmol}), \mathrm{K}_{2} \mathrm{CO}_{3}(436 \mathrm{mg}, 3.15 \mathrm{mmol})$, and $\mathrm{Bu}_{4} \mathrm{NCl}_{(349}$ $\mathrm{mg}, 1.25 \mathrm{mmol})$ in 10:1 acetonitrile- $\mathrm{H}_{2} \mathrm{O}(24 \mathrm{~mL})$ was stirred at $\mathrm{rt}$ for $15 \mathrm{~min} \mathrm{Pd}(\mathrm{OAc})_{2}$ (57 $\mathrm{mg}, 0.25$ mmol) was added, and the mixture was stirred at $60{ }^{\circ} \mathrm{C}$ for $24 \mathrm{~h}$. The solvent was removed under reduced pressure, and the resulting residue was dissolved in EtOAc $(40 \mathrm{~mL})$ and washed with saturated aqueous $\mathrm{NaHCO}_{3}$ and aqueous $\mathrm{NaCl}$ solutions. The organic phase was dried, filtered, and concentrated. Column chromatography $\left(\mathrm{Al}_{2} \mathrm{O}_{3}\right.$, hexane-EtOAc 95:5 to 75:25) of the residue afforded $\mathbf{1 0}(264 \mathrm{mg}$, $60 \%$ ) as an oil: ${ }^{1} \mathrm{H}$ NMR (300 MHz, $\left.\mathrm{CDCl}_{3}\right) \delta 2.22(\mathrm{~s}, 6 \mathrm{H}), 2.41-2.46(\mathrm{~m}, 2 \mathrm{H}), 2.56-2.63(\mathrm{~m}, 2 \mathrm{H}), 3.52$ (s, 2H), $3.66(\mathrm{~s}, 3 \mathrm{H}), 5.18(\mathrm{br}, 2 \mathrm{H}), 5.71(\mathrm{t}, J=7.2 \mathrm{~Hz}, 1 \mathrm{H}), 6.80(\mathrm{~d}, J=8.4 \mathrm{~Hz}, 1 \mathrm{H}), 7.02-7.09$ (m, 2H), 7.22-7.30 (m, 4H); ${ }^{13} \mathrm{C}$ NMR (75.4 MHz, $\left.\mathrm{CDCl}_{3}\right) \delta 28.0\left(\mathrm{CH}_{2}\right), 40.2\left(\mathrm{CH}_{2}\right), 45.4\left(2 \mathrm{CH}_{3}\right), 51.9$ $\left(\mathrm{CH}_{3}\right), 59.4\left(\mathrm{CH}_{2}\right), 70.3\left(\mathrm{CH}_{2}\right), 119.6(\mathrm{CH}), 123.8(\mathrm{C}), 125.5(\mathrm{C}), 126.1(\mathrm{CH}), 127.3(2 \mathrm{CH}), 128.9(\mathrm{CH})$, $129.8(\mathrm{CH}), 130.7(\mathrm{CH}), 131.9(\mathrm{CH}), 133.5(\mathrm{C}), 139.5(\mathrm{C}), 145.5(\mathrm{C}), 154.4(\mathrm{C}), 172.0(\mathrm{C})$; IR $(\mathrm{NaCl})$ 1225, 1489, $1739 \mathrm{~cm}^{-1}$. Anal. Calcd for $\mathrm{C}_{22} \mathrm{H}_{25} \mathrm{NO}_{3} \cdot 1 / 4 \mathrm{H}_{2} \mathrm{O}: \mathrm{C}, 74.24 ; \mathrm{H}, 7.22 ; \mathrm{N}, 3.94$. Found: C, $74.59 ; \mathrm{H}, 7.14 ; \mathrm{N}, 3.77$.

(E)-Methyl 11-[3-(Dimethylamino)propylidene]-6,11-dihydrodibenz[b,e]oxepin-2-acetate (11).

Operating as in the above preparation of 10, from $E \mathbf{- 2}(100 \mathrm{mg}, 0.21 \mathrm{mmol})$, compound 11 (40 $\mathrm{mg}$, $55 \%)$ was obtained as an oil after column chromatography $\left(\mathrm{Al}_{2} \mathrm{O}_{3}\right.$, hexane-EtOAc 95:5 to 75:25): ${ }^{1} \mathrm{H}$ 
NMR (300 MHz, $\left.\mathrm{CDCl}_{3}\right) \delta 2.16(\mathrm{~s}, 6 \mathrm{H}), 2.28-2.43(\mathrm{~m}, 4 \mathrm{H}), 3.53(\mathrm{~s}, 2 \mathrm{H}), 3.67(\mathrm{~s}, 3 \mathrm{H}), 4.78(\mathrm{br}, 1 \mathrm{H})$, 5.54 (br, 1H), $6.03(\mathrm{t}, J=6.9 \mathrm{~Hz}, 1 \mathrm{H}), 6.70(\mathrm{~d}, J=8.4 \mathrm{~Hz}, 1 \mathrm{H}), 7.20$ (dd, $J=8.5,2.2 \mathrm{~Hz}, 1 \mathrm{H}), 7.17-$ $7.37(\mathrm{~m}, 5 \mathrm{H}) ;{ }^{13} \mathrm{C}$ NMR (75.4 MHz, $\left.\mathrm{CDCl}_{3}\right) \delta 27.7\left(\mathrm{CH}_{2}\right), 40.2\left(\mathrm{CH}_{2}\right), 45.3\left(2 \mathrm{CH}_{3}\right), 52.0\left(\mathrm{CH}_{3}\right), 59.2$ $\left(\mathrm{CH}_{2}\right), 70.0\left(\mathrm{CH}_{2}\right), 119.3(\mathrm{CH}), 126.1(\mathrm{C}), 127.2(\mathrm{C}), 127.7(\mathrm{CH}), 127.8(\mathrm{CH}), 128.1(\mathrm{CH}), 128.4(\mathrm{CH})$, $129.6(\mathrm{CH}), 130.2(\mathrm{CH}), 130.7(\mathrm{CH}), 134.1(\mathrm{C}), 139.6(\mathrm{C}), 141.0(\mathrm{C}), 154.1(\mathrm{C}), 172.2(\mathrm{C})$; IR $(\mathrm{NaCl})$ 1224, 1489, $1738 \mathrm{~cm}^{-1}$. Anal. Calcd for $\mathrm{C}_{22} \mathrm{H}_{25} \mathrm{NO}_{3}$ : C, 75.19; H, 7.17; N, 3.99. Found: C, 74.82; H, $7.14 ; \mathrm{N}, 3.81$.

(Z)-11-[3-(Dimethylamino)propylidene]-6,11-dihydrodibenz[b,e]oxepin-2-acetic

Acid

Hydrochloride. $5 \mathrm{~N}$ aqueous $\mathrm{NaOH}(6.2 \mathrm{~mL}, 31.0 \mathrm{mmol})$ was added to a solution of $\mathbf{1 0}(3.32 \mathrm{~g}, 9.45$ mmol) in $\mathrm{MeOH}(105 \mathrm{~mL})$ and $\mathrm{H}_{2} \mathrm{O}(21 \mathrm{~mL})$. The mixture was stirred at $\mathrm{rt}$ for $3 \mathrm{~h}$, neutralized with $2 \mathrm{~N}$ aqueous $\mathrm{HCl}$, and concentrated to dryness. The resulting residue was dissolved in $\mathrm{H}_{2} \mathrm{O}$ and the solution was filtered through an ionic exchange resin DIAION HP-20 $\left(\mathrm{H}_{2} \mathrm{O}\right.$ until negative test of $\mathrm{AgNO}_{3}$ and then $\mathrm{MeOH}$ as eluents). The methanolic fractions gave olopatadine $(2.64 \mathrm{~g}, 83 \%) .37 \% \mathrm{HCl}(1 \mathrm{~mL}$, $12.0 \mathrm{mmol})$ was added to a stirred solution of the above olopatadine in THF $(65 \mathrm{~mL})$, and the mixture was stirred for $5 \mathrm{~min}$. The solid formed was filtered and suspended in acetone $(60 \mathrm{~mL})$. The suspension was refluxed for $30 \mathrm{~min}$, cooled, and filtered to give olopatadine hydrochloride as a solid (2.33 g, 66\% overall): mp 231-233 ${ }^{\circ} \mathrm{C}(\mathrm{dec}) ;{ }^{1} \mathrm{H}$ NMR (300 MHz, $\left.\mathrm{CD}_{3} \mathrm{OD}\right) \delta 2.86(\mathrm{~s}, 6 \mathrm{H}), 2.83-2.91(\mathrm{~m}, 2 \mathrm{H}), 3.28-$ $3.34(\mathrm{~m}, 2 \mathrm{H}), 3.57(\mathrm{~s}, 2 \mathrm{H}), 5.19(\mathrm{br}, 2 \mathrm{H}), 5.67(\mathrm{t}, J=7.3 \mathrm{~Hz}, 1 \mathrm{H}), 6.81(\mathrm{~d}, J=8.4 \mathrm{~Hz}, 1 \mathrm{H}), 7.07-7.13$ (m, 2H), 7.26-7.37 (m, 4H); ${ }^{13} \mathrm{C}$ NMR (75.4 MHz, $\left.\mathrm{CD}_{3} \mathrm{OD}\right) \delta 26.4\left(\mathrm{CH}_{2}\right), 40.5\left(\mathrm{CH}_{2}\right), 43.4\left(2 \mathrm{CH}_{3}\right), 58.0$ $\left(\mathrm{CH}_{2}\right), 71.5\left(\mathrm{CH}_{2}\right), 120.3(\mathrm{CH}), 124.8(\mathrm{C}), 126.5(\mathrm{CH}), 127.0(\mathrm{CH}), 128.4(\mathrm{C}), 128.5(\mathrm{CH}), 129.0(\mathrm{CH})$, $130.1(\mathrm{CH}), 131.7(\mathrm{CH}), 132.8(\mathrm{CH}), 135.1(\mathrm{C}), 144.5(\mathrm{C}), 145.6(\mathrm{C}), 155.9(\mathrm{C}), 175.7(\mathrm{C})$; IR $(\mathrm{KBr})$ 1225, 1491, 1716, $2927 \mathrm{~cm}^{-1}$. Anal. Calcd for $\mathrm{C}_{21} \mathrm{H}_{24} \mathrm{NClO}_{3} \cdot \mathrm{H}_{2} \mathrm{O}: \mathrm{C}, 64.36 ; \mathrm{H}, 6.69 ; \mathrm{N}, 3.57$. Found: C, 64.59; H, 6.30; N, 3.63. 
Hydrochloride. Operating as above, from ester 11 (973 $\mathrm{mg}, 2.77 \mathrm{mmol}$ ), trans-olopatadine hydrochloride was obtained as a white solid (728 mg, 70\%): $\mathrm{mp} 170-173{ }^{\circ} \mathrm{C} ;{ }^{1} \mathrm{H}$ NMR (300 MHz, $\left.\mathrm{CD}_{3} \mathrm{OD}\right) \delta 2.56-2.63(\mathrm{~m}, 2 \mathrm{H}), 2.75(\mathrm{~s}, 6 \mathrm{H}), 3.13(\mathrm{t}, J=7.6 \mathrm{~Hz}, 2 \mathrm{H}), 3.53(\mathrm{~s}, 2 \mathrm{H}), 4.78(\mathrm{br}, 1 \mathrm{H}), 5.51(\mathrm{br}$, 1H), $5.98(\mathrm{t}, J=7.2 \mathrm{~Hz}, 1 \mathrm{H}), 6.69(\mathrm{~d}, J=8.4 \mathrm{~Hz}, 1 \mathrm{H}), 7.06(\mathrm{dd}, J=8.3,2.3 \mathrm{~Hz}, 1 \mathrm{H}), 7.25-7.44(\mathrm{~m}$, 5H); ${ }^{13} \mathrm{C}$ NMR $\left(75.4 \mathrm{MHz}, \mathrm{CD}_{3} \mathrm{OD}\right) \delta 26.0\left(\mathrm{CH}_{2}\right), 40.8\left(\mathrm{CH}_{2}\right), 43.3\left(2 \mathrm{CH}_{3}\right), 57.9\left(\mathrm{CH}_{2}\right), 70.9\left(\mathrm{CH}_{2}\right)$, $120.3(\mathrm{CH}), 125.9(\mathrm{CH}), 127.6(\mathrm{C}), 128.5(\mathrm{C}), 128.6(\mathrm{CH}), 129.5(2 \mathrm{CH}), 130.0(\mathrm{CH}), 131.5(\mathrm{CH}), 132.0$ (CH), 135.8 (C), 141.3 (C), 144.2 (C), 155.6 (C), 175.7 (C); IR (KBr) 1223, 1484, 1725, $2960 \mathrm{~cm}^{-1}$. Anal. Calcd for $\mathrm{C}_{21} \mathrm{H}_{24} \mathrm{NClO}_{3} \cdot \mathrm{H}_{2} \mathrm{O}$ : C, 64.36; H, 6.69; N, 3.57. Found: C, 64.66; H, 6.47; N, 3.56 .

Acknowledgment: We thank the AGAUR, Generalitat de Catalunya, for Grant 2009-SGR-1111.

Supporting Information Available: Copies of the ${ }^{1} \mathrm{H}$ and ${ }^{13} \mathrm{C}$ NMR spectra of all compounds. This material is available free of charge via the Internet at http://pubs.acs.org.

\section{References}

(1) Yanni, J. M.; Stephens, D. J.; Miller, S. T.; Weimer, L. K.; Graff, G. Parnell, D.; Lang, L. S.; Spellman, J. M.; Brady, M. T.; Gamache, D. A. J. Ocular Pharmacol. Ther. 1996, 60, 389-400.

(2) Ohshima, E.; Otaki, S.; Sato, H.; Kumazawa, T.; Obase, H.; Ishii, A.; Ishii, H.; Ohmori, K.; Hirayama, N. J. Med. Chem. 1992, 35, 2074-2084.

(3) For a recent synthesis of olopatadine involving the stereoselective Pd-catalyzed tandem cyclization-hydride capture of an acetylenic aryl iodide, see: Nishimura, K.; Kinugawa, M. Org. Process Res. Dev. 2012, 16, 225-231.

(4) Bosch, J.; Bachs, J.; Gómez, A.; Alonso, Y.; Bessa, M. PCT Int. Appl. WO/2006/010459 A1. 
(5) For a review, see: Maryanoff, B. E.; Reitz, A. B. Chem. Rev. 1989, 89, 863-927.

(6) For reviews, see: (a) Link, J. T.; Overman, E. In Metal-catalyzed Cross-Coupling Reactions; Diedrich, F.; Stang, P. J. Ed. Wiley: Weinheim, 1998; pp. 231-269. (b) Beletskaya, I. P.; Cheprakov, A. V. Chem. Rev. 2000, 100, 3009-3066. (c) Larhed M.; Hallberg, A. In Handbook of Organopalladium Chemistry for Organic Synthesis; Negishi, E., Ed.; Wiley: New York, 2002; Vol. 1, pp. 1133-1178.

(7) Aldehyde 4 was prepared by formylation of methyl 4-hydroxyphenylacetate following a procedure (paraformaldehyde, anh. $\mathrm{MgCl}_{2}$, and triethylamine in acetonitrile) reported for the ortho-formylation of related phenols: Hofsløkken, N. U.; Sakttebøl, L. Acta Chem. Scand. 1999, 53, 258-262.

(8) For the reaction of this ylide with a hindered aldehyde to stereoselectively form a Z-olefin, see: Corey, E. J.; Desai, M. C. Tetrahedron Lett. 1985, 26, 5747-5748.

(9) Phosphonium iodide $6(\mathrm{X}=\mathrm{I})$ was prepared by reaction of 3-(dimethylamino)propyl chloride hydrochloride with $\mathrm{PPh}_{3}$ in refluxing acetonitrile in the presence of $\mathrm{NaI}$.

(10) The yields were lower when using less excess of ylide. Heating at $60{ }^{\circ} \mathrm{C}$ resulted in less clean crude reaction mixtures.

(11) Phosphonium chloride $6(\mathrm{X}=\mathrm{Cl})$ was prepared by reaction of 3-(dimethylamino)propyl chloride hydrochloride with $\mathrm{PPh}_{3}$ in refluxing 1-butanol. This procedure proved to be more satisfactory than the one previously reported: de Castro Dantas, T. N.; Laval, J. P.; Lattes, A. Phosphorus Sulfur 1982, 13, 97-105.

(12) (a) Linderman, R. J.; Meyers, A. I. Tetrahedron Lett. 1983, 24, 3043-3046. (b) Maryanoff, B. E.; Reitz, A. B.; Duhl-Emswiler, B. A. J. Am. Chem. Soc. 1985, 107, 217-226; and references cited therein. (13) Reitz, A. B.; Nortey, S. O.; Jordan, A. D.; Mutter, M. S.; Maryanoff, B. E. J. Org. Chem. 1986, 51, $3302-3308$.

(14) Zhang, X.-X.; Lippard, S. J. J. Org. Chem. 2000, 65, 5298-5305. 
(15) Pilkington, B. L.; Armstrong, S.; Barnes, N. J.; Barnett, S. P.; Clarke, E. D.; Crowley, P. J.; Fraser, T. E. M.; Hughes, D. J.; Mathews, C. J.; Salmon, R.; Smith, S.C.; Viner, R.; Whittingham, W.; Williams, J.; Whittle, A. J.; Mound, W. R.; Urch, C. J. PCT Int. Appl. WO/01/90105 A1.

(16) Tetraalkylammonium salts have been used to enhance the reactivity in Heck-type reactions: Jeffery, T. J. Chem. Soc., Chem. Commun. 1984, 1287-1289.

(17) Jeffery, T. Tetrahedron Lett. 1985, 26, 2667-2670.

(18) The addition of $\mathrm{PPh}_{3}$ resulted in more complex crude mixtures.

(19) Jeffery, T. Tetrahedron 1996, 52, 10113-10130. 